\title{
BAG3 promotes chondrosarcoma progression by upregulating the expression of $\beta$-catenin
}

\author{
HUIJUAN SHI, WENFANG CHEN, YU DONG, XIAOFANG LU, WENHUI ZHANG and LIANTANG WANG
}

Department of Pathology, The First Affiliated Hospital of Sun Yat-Sen University, Guangzhou, Guangdong 510080, P.R. China

Received April 19, 2017; Accepted February 1, 2018

DOI: $10.3892 / \mathrm{mmr} .2018 .8611$

\begin{abstract}
To investigate the roles of B-cell lymphoma-2 associated athanogene 3 (BAG3) in human chondrosarcoma and the potential mechanisms, the expression levels of BAG3 were detected in the present study, and the associations between BAG3 and clinical pathological parameters, clinical stage as well as the survival of patients were analyzed. The present study detected BAG3 mRNA and protein expression in the normal cartilage cell line HC-a and in SW1353 chondrosarcoma cells by reverse transcription-quantitative polymerase chain reaction and western blot analysis. The BAG3 protein expression in 59 cases of chondrosarcoma, 30 patients with endogenous chondroma and 8 cases of normal cartilage was semi-quantitatively analyzed using the immunohistochemical method. In addition, the BAG3 protein expression level, the clinical pathological parameters, clinical stage and the survival time of patients with chondrosarcoma were analyzed. The plasmid transfection method was employed to upregulate the expression BAG3 and small RNA interference to downregulate the expression of BAG3 in SW1353 cells. The expression levels of BAG3 protein and mRNA were significantly increased in the chondrosarcoma cell line when compared with the normal cartilage cell line. The immunohistochemistry results indicated that BAG3 protein was overexpressed in the tissue of human chondrosarcoma. Statistical analysis showed that the expression level of BAG3 was significantly increased in the different Enneking staging of patients with chondrosarcoma and Tumor staging, and there were no statistical differences in age, gender, histological classification and tumor size. In the in vitro experiments, the data revealed that BAG3 significantly promoted chondrosarcoma cell proliferation, colony-formation, migration and invasion;
\end{abstract}

Correspondence to: Professor Liantang Wang, Department of Pathology, The First Affiliated Hospital of Sun Yat-Sen University, 58 Zhongshan Erlu, Dongshan, Guangzhou, Guangdong 510080, P.R. China

E-mail:shihj@mail.sysu.edu.cn

Key words: B-cell lymphoma-2 associated athanogene 3, chondrosarcoma, $\beta$-catenin, epithelial to mesenchymal transition however, it inhibited chondrosarcoma cell apoptosis. It was observed that BAG3 upregulated $\beta$-catenin expression at the mRNA and protein levels. In addition, BAG3 induced the expression of runt-related transcription factor 2 (RUNX2) in chondrosarcoma cells by upregulating $\beta$-catenin. These clinical analyses revealed a positive association between $\beta$-catenin and BAG3 in chondrosarcoma tumors. BAG3 was significantly increased in chondrosarcoma cells and tissues compared with the normal cartilage cells, tissue and cartilage benign tumors. Thus, BAG3 may serve as an oncogene in the development of chondrosarcoma via the induction of RUNX2 expression. The results of the present study contribute to further research on the biological development of chondrosarcoma.

\section{Introduction}

Chondrosarcoma is a malignant cartilage-forming cancer, which is the most common primary malignant bone tumor in adults $(1,2)$. Currently, wide local excision is the most effective treatment since the treatment of patients with chondrosarcomas displays barely response to both chemotherapy and radiation therapy $(3,4)$. Moreover, most chemotherapy drugs for chondrosarcoma are associated with strong toxicities for normal tissues (5). Thus, effective therapeutic approaches to improve chondrosarcoma clinical outcome are still under investigation.

B-cell lymphoma-2 associated athanogene 3 (BAG3) is a member of BAG family of co-chaperones (6). It has a modular structure that contains a BAG domain, a WW domain, a proline-rich (PxxP) domain interacting with proteins (7). In addition, the WW domains connect BAG3 to SH3 domains of its binding proteins (7). The functions of BAG3 in cancers have been described. BAG3 protein is known to interact with the ATPase domain of the heat shock protein (Hsp)70 through BAG domain $(8,9)$. A study reported that the Hsp70-BAG3 interactions regulate multiple cancer-related signaling networks (9). It has illustrated that BAG3 interacts with the SH3 domain of Src, thereby mediating the effects of Hsp70 on Src signaling (9). BAG3 has been reported to associate with GRP78, resulting in sensitization of cancer cells to DNA damaging agents (10). Moreover, it has been demonstrated that inhibition of endogenous BAG3 by siRNA could enhance the effectiveness of chemotherapy (11), suggesting that BAG3 has the potential to be a therapeutic target of human malignancies. However, the roles of BAG3 in chondrosarcoma remain unclear. 
The potential roles and mechanisms of the BAG3 in modulation of chondrosarcoma malignancies have been largely unknown previously. In this study, we investigated the functions of BAG3 in human chondrosarcoma by comparison of the expressions of BAG3 in normal cartilage tissue, benign chondroma and chondrosarcoma. Meanwhile, we assessed the roles of BAG3 in the proliferation and migration of chondrosarcoma cells. Our study identified the potential roles of BAG3 in chondrosarcoma, which will contribute to the development of novel therapeutics for the treatments of chondrosarcoma patients.

\section{Materials and methods}

Patient specimens and information. Fifty-nine cases of chondrosarcoma, thirty cases of naturally express cartilage tumors and eight cases of normal cartilage tissues were randomly obtained from the Department of Pathology at the First Affiliated Hospital of Sun Yat-Sen University (Guangzhou, China) between January 2006 and November 2015. The chondrosarcoma tissues were from 33 males and 26 females. There are thirty-two cases under the age of forty-three years and twenty-seven cases over the age of forty-three years. Moreover, according to the Enneking surgical staging for musculoskeletal neoplasms classification, there are 30 cases belong to stage I, 25 cases belong to stage II and four cases belong to stage III. According to WHO histology grading: There were 31 cases in grade I, 22 cases in grade II III and 6 cases of undifferentiated. Patients with preoperative radiotherapy or chemotherapy were excluded. In addition, normal cartilage tissues were obtained at $2-5 \mathrm{~cm}$ away from the tumors. The material had been fixed in $4 \%$ buffered formalin and embedded in paraffin. Prior to the research, patient's written informed consent and approval from the Institute Research Ethics Committee of The First Affiliated Hospital of Sun Yat-Sen University was obtained, and the Ethics Committee approval number is 201301.

Cell culture. The human chondrosarcoma cell line SW1353 was purchased from the Type Culture Collection of the Chinese Academy of Sciences (Shanghai, China). The normal chondrocyte cell line HC-a was purchased from Shanghai Yu Bo Biological Technology Co., Ltd. (Shanghai, China). SW1353 cells were cultivated in Dulbecco's modified Eagle's medium (DMEM) supplemented with 10\% heat-inactivated fetal bovine serum (FBS). HC-a cells were cultured in RPMI-1640 supplemented with $10 \%$ FBS. All cultures contained $1 \%$ penicillin/streptomycin and were incubated in an incubator with $5 \% \mathrm{CO}_{2}$ at $37^{\circ} \mathrm{C}$.

Antibodies and reagents. The antibodies were purchased from: Rabbit polyclonal Anti-BAG3 antibody (cat. no. ab47124; Abcam, Cambridge, UK); rabbit polyclonal anti-RUNX2 antibody (cat. no. ab28931; Abcam); Rabbit monoclonal Anti-GAPDH antibody (cat. no. 2118; Cell Signaling Technology, Inc., Danvers, MA, USA) and $\beta$-catenin were purchased form the epithelial-mesenchymal transition (EMT) Antibody Sampler kit (cat. no. 9782; Cell Signaling Technology, Inc.).

Plasmid DNA and siRNA transfections. Expression vector containing wild type BAG3 were constructed from pcDNA3.1 according to the previous report (10). The vector was double digested at $\mathrm{XhoI}$ and $\mathrm{BamHI}$ sites. SiRNA oligonucleotides for BAG3 was purchased from Invitrogen with a scrambled siRNA as a control. The siBAG3 sequences were described as following: sense: 5'-AAGGUUCAGACCAUCUUGGAA-3'; antisense: 5'-TTCCGTGGTCTGCCTT-3'. Cells were transfected using the Lipofectamine ${ }^{\circledR} 2000$ Transfection reagent (Invitrogen) according to the manufacturer's protocol. siRNA transfection was performed with $100 \mathrm{nmol} / 1$ and plasmid DNA was transfected with $2 \mu \mathrm{g}$. At $48 \mathrm{~h}$ after transfection, whole-cell lysates were prepared for further analysis.

Polymerase chain reaction $(P C R)$ and reverse transcription-quantitative PCR (RT-qPCR). Total RNA was extracted after homogenization of cells and tissues using RNeasy mini kit (Qiagen Sciences, Inc., Germantown, MD, USA). Total RNA $(1 \mu \mathrm{g})$ was reversely transcribed with the High Capacity cDNA Reverse Transcription kit (Applied Biosystems; Thermo Fisher Scientific, Inc., Waltham, MA, USA). The cDNA reaction was diluted to 1:10 for use as template for RT-qPCR. TaqMan Gene Expression Assays primers and probes specific to BAG3 or $\beta$-catenin were used for expression analysis and $18 \mathrm{~S}$ ribosomal primers and probes (Applied Biosystems; Thermo Fisher Scientific, Inc.) were used as internal controls. PCR amplifications were performed in a final reaction volume of $10 \mu \mathrm{l}$ containing, $5.5 \mu \mathrm{l}$ of TaqMan Universal PCR Master Mix (Applied Biosystems; Thermo Fisher Scientific, Inc.), $0.5 \mu \mathrm{l}$ of the primers and probes mix and $4.5 \mu \mathrm{g}$ of the cDNA diluted solution. The primers for qPCR are: BAG3-forward: 5'-TGG GAGATCAAGATCGACCC-3'; BAG3-reverse: 5'-GGGCCA TTGGCAGAGGATG-3'. All qPCR reactions were carried out in triplicate and repeated at least twice. The $\triangle \mathrm{Cq}$ for mRNA expression was calculated relative to the $\mathrm{Cq}$ (quantitation cycle) of $18 \mathrm{~S}$ ribosomal RNA. Relative mRNA expression was calculated using the formula $2^{-\Delta \Delta \mathrm{Cq}}$.

Cell growth assay. For the measurements of cell growth, a total of $2.5 \times 10^{3}$ cells/well were seeded in 24-well plates. Cells were transfected with either control siRNA, siBAG3 or overexpression vector of BAG3 for $48 \mathrm{~h}$. Cell growth was determined by the 3-(4,5 dimethylthiazol-2-yl)-2,5-diphenyltetrazolium bromide (MTT) assay according to the previous report (10). Absorbance was measured spectrophotometrically at $490 \mathrm{~nm}$ by using the Universal Microplate Reader EL800 (BioTek Instruments, Inc., Winooski, VT, USA). All experiments were performed in triplicate.

Apoptosis assay. Cell apoptosis assay was performed using Apoptosis kit with Annexin V FITC and PI from Invitrogen (Thermo Fisher Scientific, Inc.) according to the manufacturer's instructions. Briefly, cells were transfected with either control siRNA, siBAG3 or overexpression vector of BAG3 for $48 \mathrm{~h}$, cells were trypsinized and $1-5 \times 10^{6}$ cells of each group were collected for the apoptosis assay. After staining with Annexin V-FITC and PI, samples were analyzed by using fluorescence-activated cell scanner (FACScan) flow cytometer (BD Biosciences, Franklin Lakes, NJ, USA).

Clonogenic assay. SW1353 cells were transfected with either control siRNA, siBAG3 or overexpression vector of BAG3 for 
A

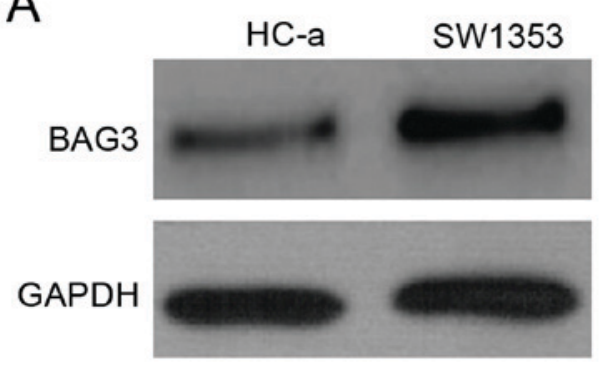

B
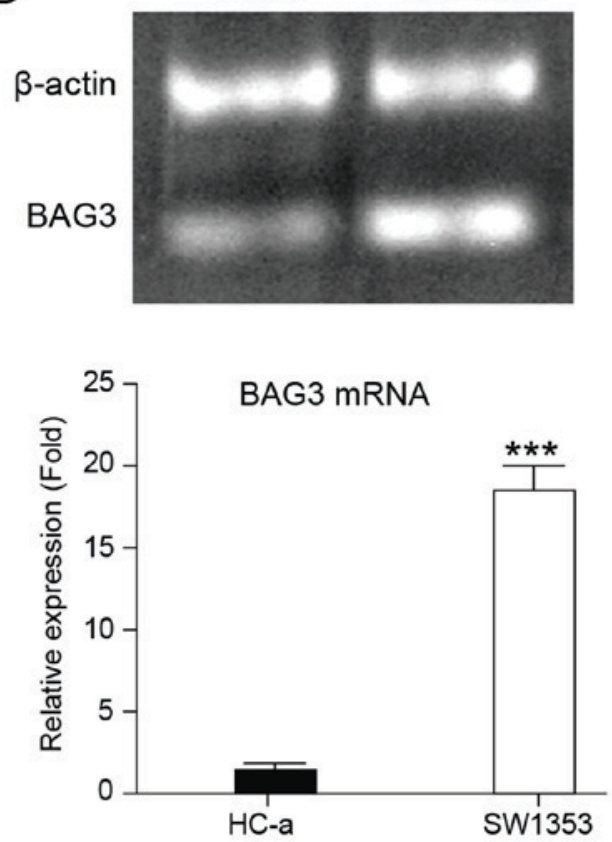

C

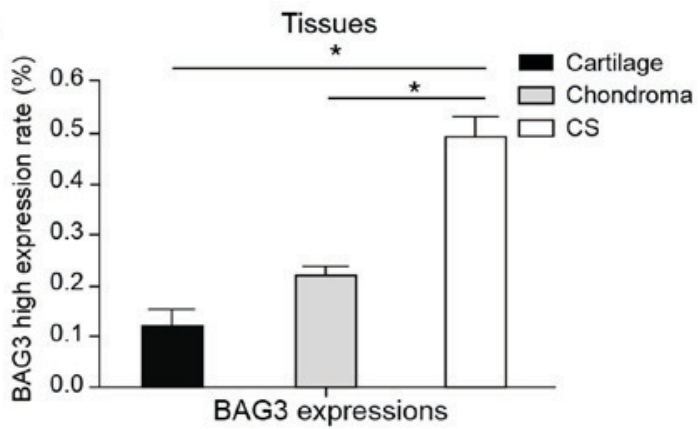

D

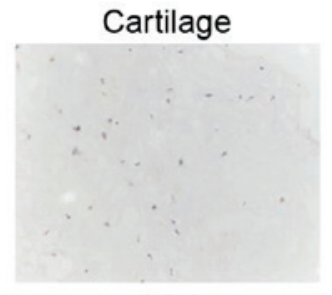

CS I

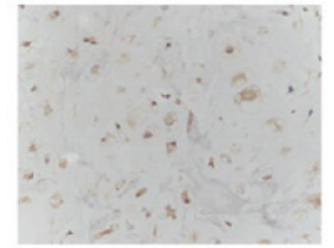
CS III

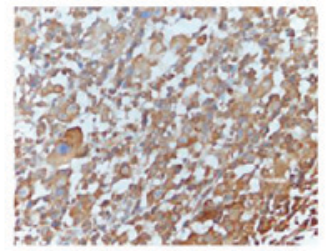

Chondroma

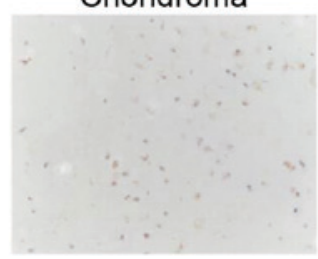

CS II

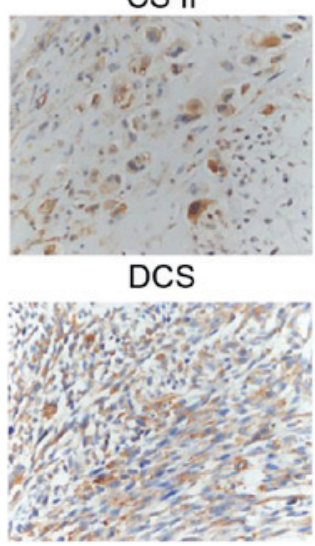

Figure 1. Upregulation of BAG3 in human chondrosarcoma. (A) The protein expression of BAG3 in the SW1353 chondrosarcoma cell line and the normal chondrocyte cell line, HC-a by western blotting; GAPDH was used as a loading control. (B) The mRNA expression of BAG3 in the SW1353 chondrosarcoma cell line and normal chondrocyte cell line, HC-a by RT-qPCR; $\beta$-actin was used as a loading control. (C) The mRNA expression of BAG3 in human cartilage tissues, chondroma tissues and chondrosarcoma tumors by RT-qPCR. (D) Immunohistochemical staining for BAG3 expression in human cartilage tissues, chondroma tissues, chondrosarcoma tumors from grade I to III and DCS. The micrographs shown represent the range of staining observed in tissues (magnification, $\mathrm{x} 200$ ). Data are presented as the mean \pm standard error of three independent experiments. "P<0.05, as indicated; ${ }^{* * * *} \mathrm{P}<0.001 \mathrm{vs}$. HC-a. BAG3, B-cell lymphoma-2 associated athanogene 3; DCS, dedifferentiated chondrosarcoma; RT-qPCR, reverse transcription-quantitative polymerase chain reaction; CS, chondrosarcoma.

$48 \mathrm{~h}, 1 \times 10^{3}$ cells were seeded on $10 \mathrm{~cm}$ dish with regular cell culture medium for two weeks and the colonies were stained with gentian violet after methanol fixation, and visible colonies (>50 cells) were counted. Colonies from randomly-selected image areas of three replicate wells were enumerated.

Cell migration assay. The cell migration assay was performed using Transwell cell culture inserts (Invitrogen; Thermo Fisher Scientific, Inc.). The cells were transfected with control siRNA, siBAG3 or overexpression vector of BAG3 for $48 \mathrm{~h}$ then cells were trypsinized and $5 \times 10^{3}$ cells of each group were plated to transwell insert to migrate for $24 \mathrm{~h}$. The passaged cells were stained with crystal violet solution, and absorbance was measured at $595 \mathrm{~nm}$. In the wound-healing assays, cell motility was assessed by measuring the movement of cells toward the scratch. The speed of wound closure was monitored after $24 \mathrm{~h}$ by measuring the ratio of the distance of the wound at $0 \mathrm{~h}$. Each experiment was performed in triplicate.
Immunohistochemistry. For IHC, the tissue samples were cut in 4-micro meter sections. After antigen retrieval, the sections were incubated with anti-BAG3 or anti- $\beta$-catenin antibodies at $4^{\circ} \mathrm{C}$ overnight, followed by a HRP-labeled second antibody. The staining was photographed under an inverted light microscope (Olympus, Tokyo, Japan). The scoring criteria for staining were as follows: staining was scored by integrating the staining intensity of positive cells (trichotomy) and the percentage of positive cells in the tumor area (quartering). The scoring criteria for staining intensity were regarded as: 0 , no staining; 1 , light yellow staining; 2 , yellow staining; and 3 , brown staining. Areas: 0 , no staining; $1, \leq 10 \% ; 2,10-25 \% ; 3,25-50 \%$; and 4 , $>50 \%$. Two scores were multiplied to obtain a score of 0 to 12 , setting 6 as the threshold by the log-rank test. Low expression, score $<6$; high expression score $\geq 6$. $\beta$-catenin was judged by the Maruyama standard (12) and mainly expressed in the cell membrane. The expression of membrane was normal when $\geq 70 \%$ and decreased when $<70 \%$, respectively. The expression 
of membrane was decreased and abnormal when $\geq 10 \%$ plasma or nuclear expression. Immunohistochemical results were independently judged by three pathologists, and disagreeable immunohistochemical results were finally decided after discussion.

Western blotting. Cells were lysed with RIPA buffer (Thermo Fisher Scientific, Inc.). The concentration of samples was measured using the BCA kit (Pierce; Thermo Fisher Scientific, Inc.) as instructed. The lysates were separated by $10 \%$ SDS-PAGE gels, and polyvinylidenefluoride (PVDF) membranes (EMD Millipore, Billerica, MA, USA) were used for protein transfer. The PVDF membranes were then blocked by $5 \%$ skim milk containing $0.1 \%$ Tween-20 at room temperature for $1 \mathrm{~h}$, and then incubated over night at $4^{\circ} \mathrm{C}$ with primary antibody $(1: 1,000)$. After washing with PBST the following day, the membranes were incubated with secondary antibodies (1:5,000; Invitrogen; Thermo Fisher Scientific, Inc.) at room temperature for $1 \mathrm{~h}$. Then, bands were captured using BeyoECL Plus (Beyotime Institute of Biotechnology, Haimen, China) and detected using a BioImaging System. The protein expression criteria referred to previous studies (12).

Statistical analysis. Data were analyzed using the Statistical Package for the Social Sciences (SPSS), version 18 (SPSS, Inc., Chicago, IL, USA). The Pearson's Chi-square test was used to analyze the relationship between BAG3 expression and clinical pathological characteristics. One-way analysis of variance (ANOVA), Student's t-test, or Chi-square tests were used to compare differences between groups. $\mathrm{P}<0.05$ was considered to indicate a statistically significant difference.

\section{Results}

BAG3 was significantly upregulated in human chondrosarcoma. Multiple studies have identified functions of BAG3 which is correlated with malignant phenotypes of cancers. To uncover the roles of BAG3 in human chondrosarcoma, we sought to compare the expression levels of BAG3 in human chondrosarcoma cell lines, SW1353 and normal chondrocyte cell line, HC-a. As we expected, BAG3 was significantly upregulated in chondrosarcoma cells at protein and mRNA levels (Fig. 1A and B). To evaluate whether BAG3 is a potential oncogene in human chondrosarcoma tissues, we measured the mRNA levels of BAG3 in normal human cartilage tissue, benign cartilaginous tumor chondroma and chondrosarcoma tissues. Consistently, the expression of BAG3 in chondrosarcoma patient tumor samples was significantly upregulated compared with normal human cartilage and chondroma tissues (Fig. 1C). In addition, we observed that the expressions of BAG3 in human chondrosarcoma were elevated according to the tumor malignant stages (Fig. 1D, Table I), indicating that the upregulated BAG3 expression might be the target for anti-chondrosarcoma therapy.

BAG3 promoted chondrosarcoma cells growth, migration and inhibited cells apoptosis. Our above results demonstrated that BAG3 might possess onco-protein functions. We next investigated the role of BAG3 in chondrosarcoma cell proliferation and tumor growth. SW1353 cells were transfected with siRNA
Table I. Demographic characteristics of the 59 patients with chondrosarcoma.

\begin{tabular}{|c|c|c|c|c|}
\hline \multirow[b]{2}{*}{ Characteristics } & \multicolumn{2}{|c|}{ BAG3 } & \multirow[b]{2}{*}{ Chi-square } & \multirow[b]{2}{*}{ P-value } \\
\hline & $\begin{array}{c}\text { Low } \\
\text { expression }\end{array}$ & $\begin{array}{c}\text { High } \\
\text { expression }\end{array}$ & & \\
\hline Age (years) & & & 0.817 & 0.366 \\
\hline$\leq 42$ & 18 & 14 & & \\
\hline$>42$ & 12 & 15 & & \\
\hline Sex & & & 0.410 & 0.522 \\
\hline Male & 18 & 15 & & \\
\hline Female & 12 & 14 & & \\
\hline WHO histology & & & 2.958 & 0.228 \\
\hline I & 19 & 12 & & \\
\hline II-III & 9 & 13 & & \\
\hline D- & 2 & 4 & & \\
\hline Enneking stage & & & 13.926 & 0.001 \\
\hline 1 & 23 & 7 & & \\
\hline 2 & 7 & 18 & & \\
\hline 3 & 0 & 4 & & \\
\hline T classification & & & 4.094 & 0.043 \\
\hline 1 & 8 & 2 & & \\
\hline 2 & 22 & 27 & & \\
\hline Distant & & & & \\
\hline metastasis & & & 3.270 & 0.071 \\
\hline 0 & 30 & 26 & & \\
\hline 1 & 0 & 3 & & \\
\hline
\end{tabular}

Six was set as the critical value for BAG3 staining score. High expression group, BAG3 expression value $\geq 6$; low expression group, BAG3 expression value $<6$. BAG3, B-cell lymphoma-2 associated athanogene 3; WHO, World Health Organization.

to knock down BAG3 or overexpression vector of BAG3 (Fig. 2A and B). MTT assays results indicated that knockdown BAG3 resulted in the inhibition of cell proliferation compared with the control siRNA-transfected cells (Fig. 2C). In contrast, overexpression of BAG3 promoted the cell proliferation rates (Fig. 2D). To further confirm the role of BAG3 in chondrosarcoma cell growth in vitro, we performed clonogenic assays. Knock down of BAG3 suppressed colony formation (Fig. 2E) and overexpression of BAG3 notably increased colony formation in fourteen days (Fig. 2F), suggesting BAG3 promoted chondrosarcoma cell proliferation. We evaluated the roles of BAG3 in cell apoptosis. Consistently, results in Fig. 3A and B revealed that BAG3 is required to prevent chondrosarcoma cells apoptosis. To investigate the effect of BAG3 on the viability of chondrosarcoma cells, we counted the cell number in colony formation experiment (Fig. $2 \mathrm{G}$ and $\mathrm{H}$ ) and evaluated the apoptosis rate of chondrosarcoma cells in apoptosis experiment (Fig. 3C and D).

BAG3 promotes chondrosarcoma cells migration (Fig.4A and B). The number of migration cells in the control siRNA and BAG3 siRNA were 12 and 73, respectively. On the contrary, 
A

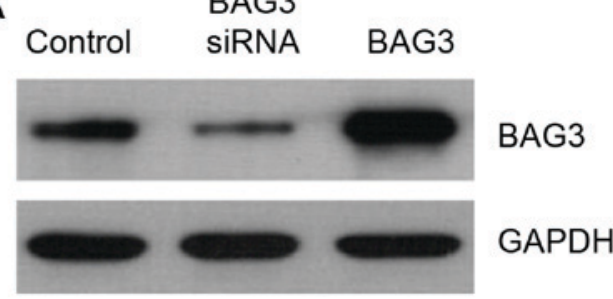

C

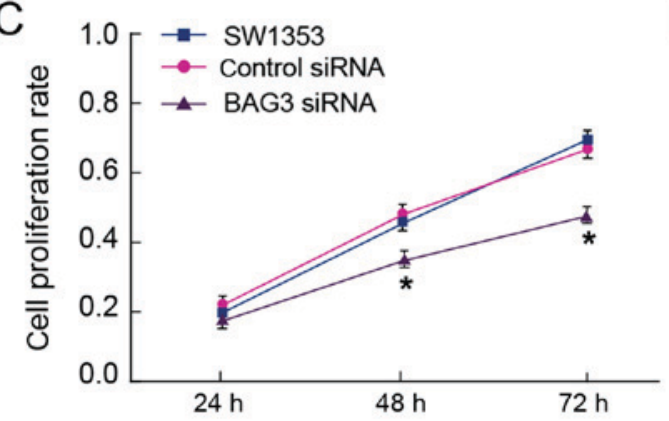

E

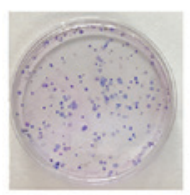

SW1353

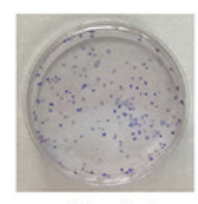

Control siRNA

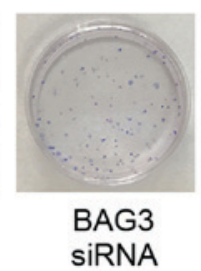

G

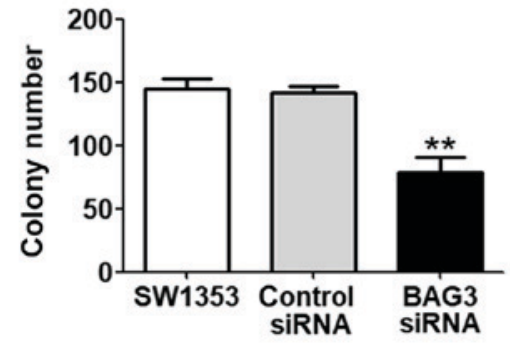

B

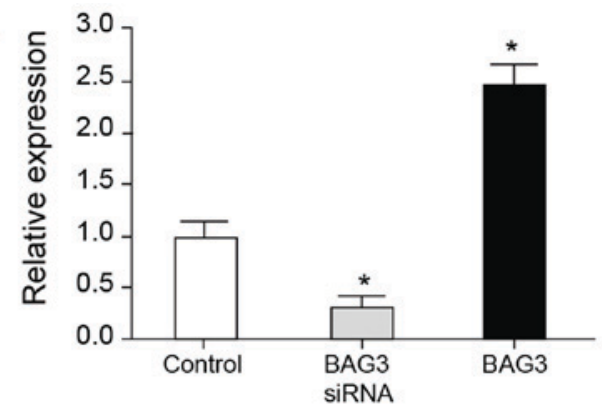

$\mathrm{D}$

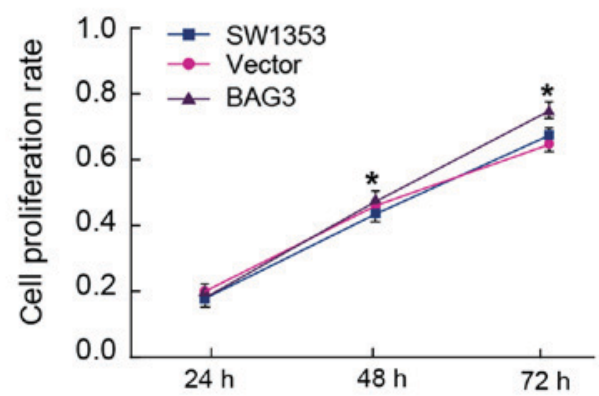

$\mathrm{F}$

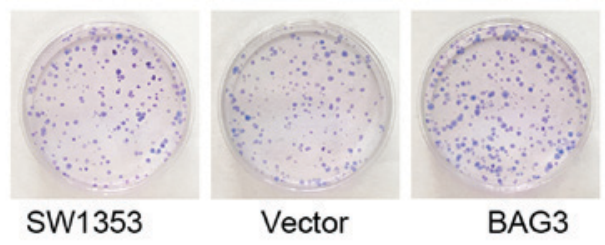

$\mathrm{H}$

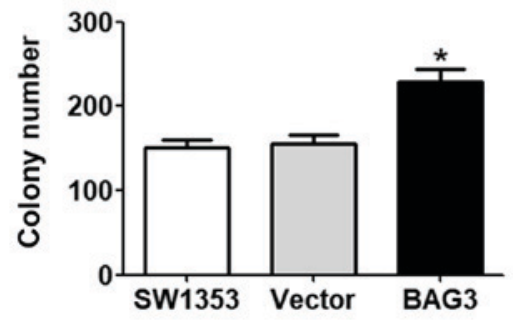

Figure 2. BAG3 promoted chondrosarcoma cell growth. (A) SW1353 cells were transfected with the control vector, BAG3 siRNA or BAG3 overexpression vectors for $48 \mathrm{~h}$, followed by western blot analysis; GAPDH was used as a loading control. (B) SW1353 cells were transfected with the control vector, BAG3 siRNA or BAG3 overexpression vectors for $48 \mathrm{~h}$, followed by mRNA expression analysis using reverse transcription-quantitative polymerase chain reaction; $\beta$-actin was used as an internal control. (C) SW1353 cells were transfected with control siRNA or BAG3 siRNA for $48 \mathrm{~h}$. The cell growth rates were measured by MTT assay. (D) SW1353 cells with transfection of control vector or BAG3 overexpression vector for $48 \mathrm{~h}$. The cell growth rates were measured by MTT assay. (E) SW1353 cells with transfection of control siRNA or BAG3 siRNA for $48 \mathrm{~h}$, followed by the analysis of colony formation. (F) SW1353 cells with transfection of control vector or BAG3 overexpression vector for $48 \mathrm{~h}$, followed by the analysis of colony formation. The SW1353 colony number following $(\mathrm{G})$ siRNA or $(\mathrm{H})$ vector transfection. Data are presented as the mean \pm standard error of three independent experiments. ${ }^{*} \mathrm{P}<0.05$ and ${ }^{* *} \mathrm{P}<0.005$ vs. control/vector. BAG3, B-cell lymphoma-2 associated athanogene 3; siRNA, small interfering RNA.

the number of migration cells in the control vector and BAG3 overexpression vector were 112 and 255, respectively (Fig. 4C). The maximum migration distance of BAG3 siRNA was $2,189 \mu \mathrm{m}$, the distance in the control siRNA and the control vector group as well as the control group were 568, 578 and $523 \mu \mathrm{m}$, respectively, and the minimum distance of BAG3 overexpression vector was $300 \mu \mathrm{m}$ (Fig. 4D).

The RUNX2 in chondrosarcoma cells were upregulated by $B A G 3$. RUNX2 was a member of the Run-related transcription factor family and is a key factor of osteoblast differentiation $(13,14)$. Studies found that mutations in the RUNX 2 gene were closely related to bone formation in mouse model and human body. In this study, we examined the effect of BAG3 on the expression of RUNX2 gene. It was found that the expression level of RUNX2 was significantly decreased after silencing BAG3 compared with the control group and increased after overexpressing BAG3 compared with the blank load group (Fig. 5A and B), indicating that BAG3 could upregulate the expression of RUNX2, as well as $\beta$-Catenin. The relative expression levels of $\beta$-Catenin and RUNX2 proteins also showed consistent results (Fig. 5C and D).

BAG3 promoted the expression of RUNX2 through upregulation of $\beta$-catenin. To further investigate signaling pathways that involve in the BAG3-promoted the expression of RUNX2 

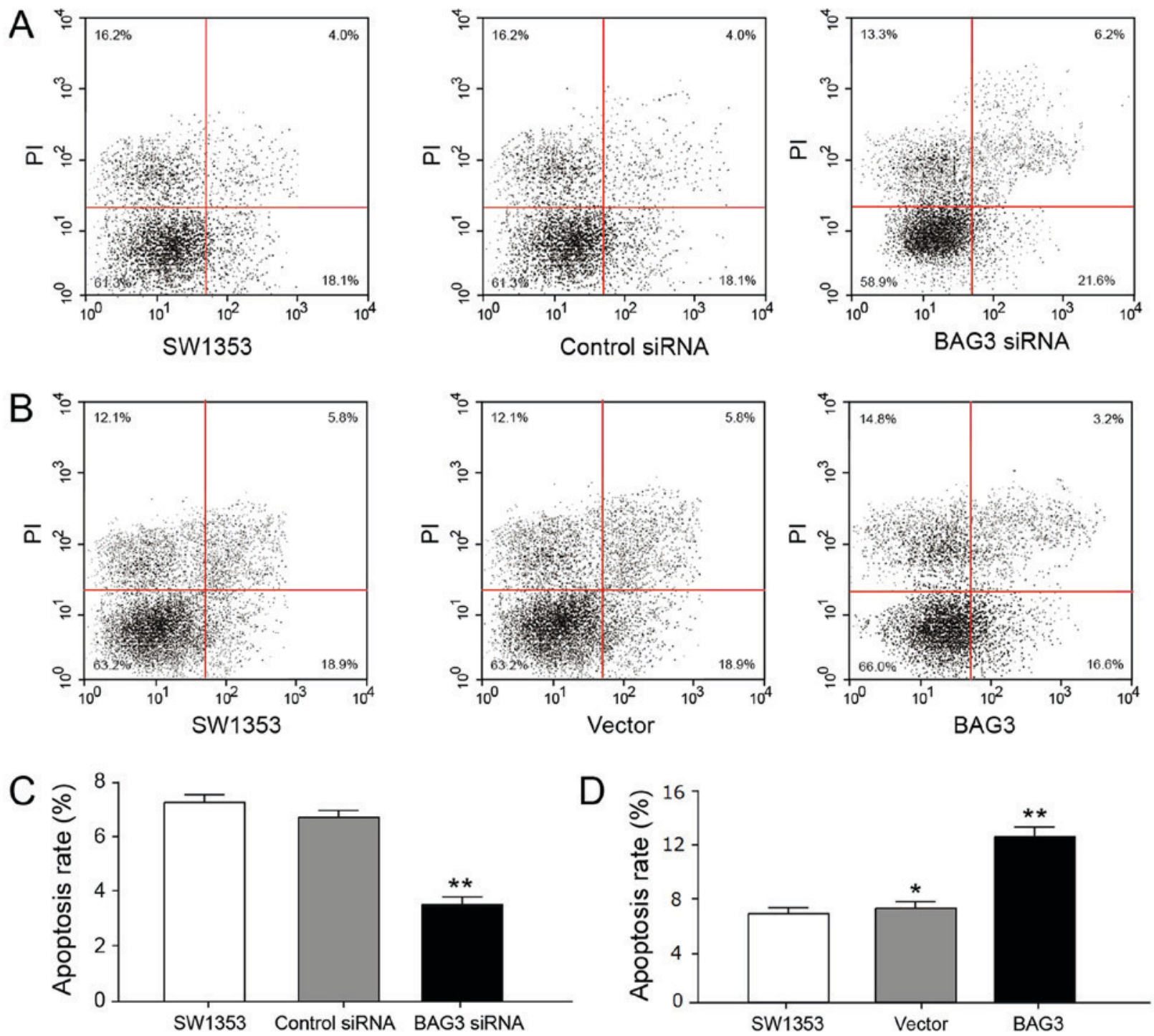

Figure 3. BAG3 inhibited chondrosarcoma cell apoptosis. The SW1353 cells were transfected with (A) control siRNA, BAG3 siRNA, (B) control vector or BAG3 overexpression vector for $48 \mathrm{~h}$, followed by analysis of apoptosis using PI/FITC staining. The SW1353 apoptotic rate of (C) control siRNA, BAG3 siRNA, (D) vector and BAG3. Data are presented as the mean \pm standard error of three independent experiments. " $\mathrm{P}<0.05$ and ${ }^{* *} \mathrm{P}<0.005$ vs. SW1353 control. BAG3, B-cell lymphoma-2 associated athanogene 3; PI, propidium iodide; FITC, fluorescein isothiocyanate; siRNA, small interfering RNA.

through upregulation of $\beta$-catenin in chondrosarcoma cells, we measured the $\beta$-catenin expression in response to BAG3 knock down or overexpression in SW1353 cells. Our results in Fig. 6A demonstrated that BAG3 upregulated both protein and mRNA levels of $\beta$-catenin, intriguing us to investigate whether BAG3 promoted the expression of RUNX2 through the upregulation of $\beta$-catenin. SW1353 cells were transfected with control vector, $\beta$-catenin siRNA or $\beta$-catenin siRNA plus BAG3 overexpressing vector. As we expected, overexpression of BAG3 could not rescue the $\beta$-catenin expression in $\beta$-catenin knocking down cells (Fig. 6B), indicating $\beta$-catenin was a downstream signal molecule of BAG3. Moreover, knock down of $\beta$-catenin significantly impeded the expression of RUNX2, which could not be rescued by overexpression of BAG3 (Fig. 6B), suggesting that BAG3promoted the expression of RUNX2 through the BAG3 downstream effector, $\beta$-catenin.

An obviously positive correlation between BAG3 expression and $\beta$-catenin in chondrosarcoma tissues. To evaluate the clinical significance of the BAG3-regulated $\beta$-catenin expression in chondrosarcoma, we first compared the expressions of $\beta$-catenin in normal chondrocyte, HC-a and chondrosarcoma cell line, SW1353. We observed a significantly upregulation of $\beta$-catenin in tumor cells (Fig. 7A and B). The expression of $\beta$-catenin were upregulated in chondrosarcoma tissues compared with normal cartilage and chondroma tissues (Fig. 7C). Moreover, the expression of $\beta$-catenin was positively correlated with the malignant stages (CS I-III and DCS) of chondrosarcoma tissues in immunohistochemistry stating (Fig. 7D), which was consistent with previous reports that $\beta$-catenin acted as an oncoprotein in human cancers (15). We next evaluated whether there is positive correlation between BAG3 and $\beta$-catenin in chondrosarcoma patient specimens. We analyzed twenty chondrosarcoma tissues, and it was observed that BAG3 was correlatively expressed with $\beta$-catenin $\left(\mathrm{P}<0.0001, \mathrm{r}^{2}=0.5748\right)$ (Fig. 7E). Meanwhile, it was found that in BAG3 low-expressed chondrosarcoma tissues, $\beta$-catenin was at lower potential to be dysregulated (40\%) 


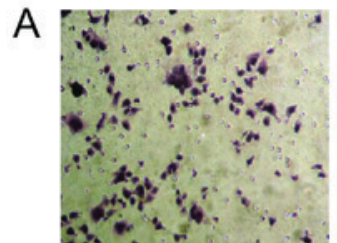

B
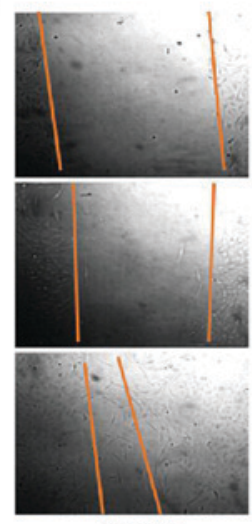

Control

C

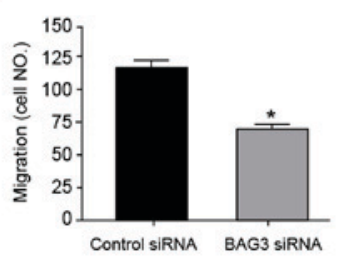

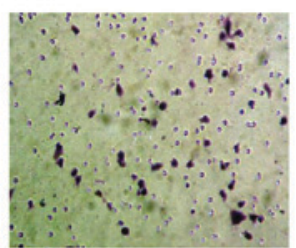

BAG3 siRNA

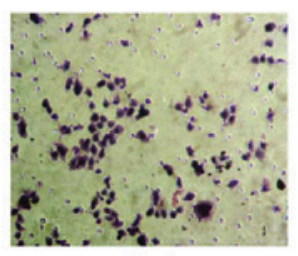

Vector

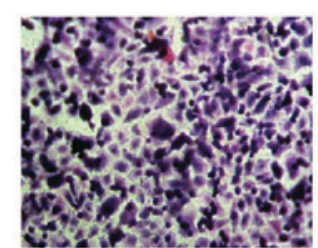

BAG3

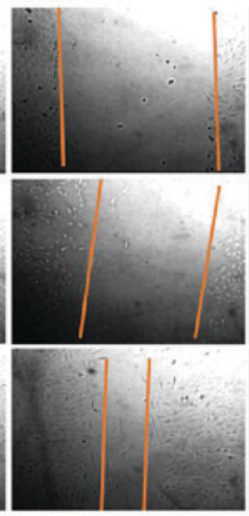

Control siRNA

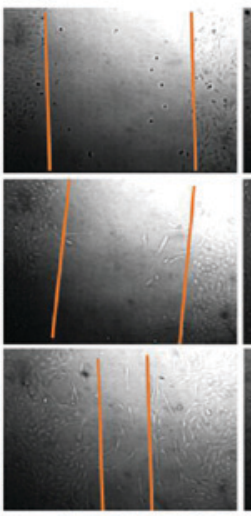

Vector

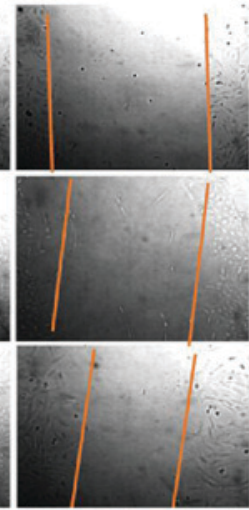

BAG3 SIRNA
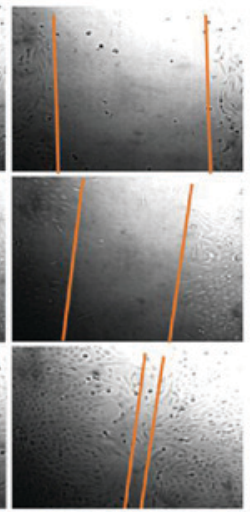

BAG3
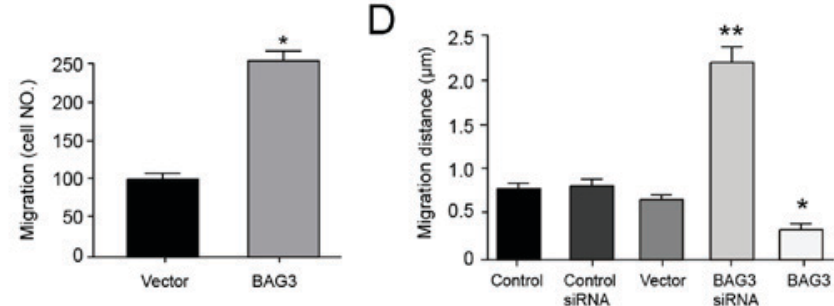

Figure 4. BAG3 promoted chondrosarcoma cell migration and invasion. (A) SW1353 cells were transfected with control siRNA, BAG3 siRNA, control vector or BAG3 overexpression vector for $48 \mathrm{~h}$, which was followed by measurements of cell invasion. (B) Migration in SW1353 cells with or without transfection of control, control siRNA, vector, BAG3 siRNA or BAG3 overexpression vector for $48 \mathrm{~h}$ (magnification, x100). (C) The number of migratory SW1353 cells (D) The migration distance of the SW1353, control siRNA, BAG3 siRNA, vector or BAG3 groups. Data are presented as the mean \pm standard error of three independent experiments. ${ }^{*} \mathrm{P}<0.05$ and ${ }^{* *} \mathrm{P}<0.005$ vs. control. BAG3, B-cell lymphoma-2 associated athanogene 3 ; siRNA, small interfering RNA.
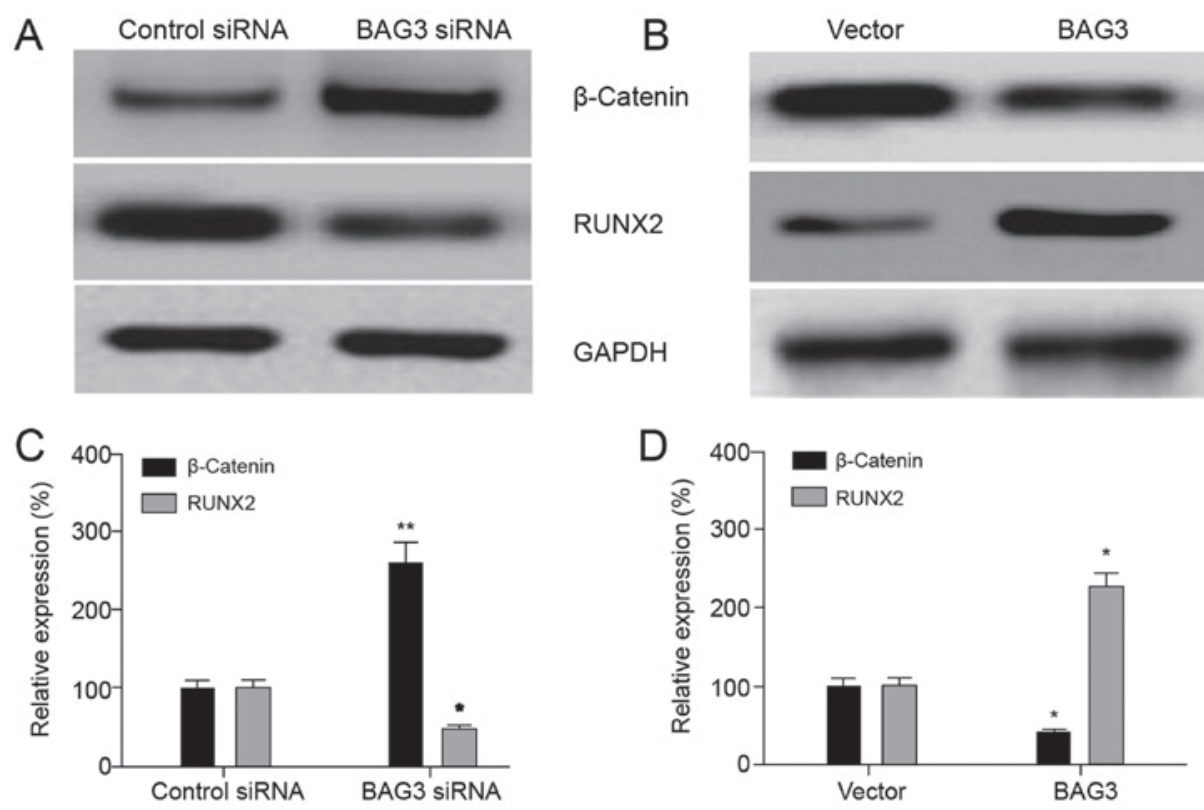

Figure 5. BAG3 upregulated RUNX2 in chondrosarcoma cells. (A) SW1353 cells were transfected with control siRNA, BAG3 siRNA, control vector or BAG3 overexpression vector for $48 \mathrm{~h}$; (B) the protein expression of $\beta$-catenin and RUNX2 was then determined by western blot analysis. GAPDH was used as a loading control. (C and D) SW1353 cells were transfected with control siRNA, BAG3 siRNA, control vector or BAG3 overexpression vector for 48 h; the relative mRNA expressions of $\beta$-catenin and RUNX2 were then determined by reverse transcription-quantitative polymerase chain reaction. $\beta$-actin was used as an internal control. Data are presented as the mean \pm standard error of three independent experiments. " $\mathrm{P}<0.05$ and ${ }^{* * *} \mathrm{P}<0.005$ vs. control. BAG3, B-cell lymphoma-2 associated athanogene 3; siRNA, small interfering RNA; RUNX2, runt-related transcription factor 2. 
A

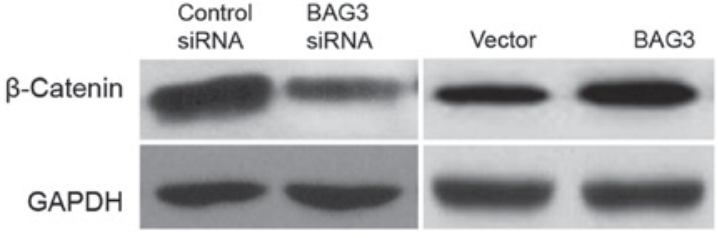

B

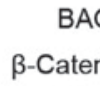

$\beta$-Catenin siRNA

BAG3

$\beta-$ Catenin

RUNX2

GAPDH
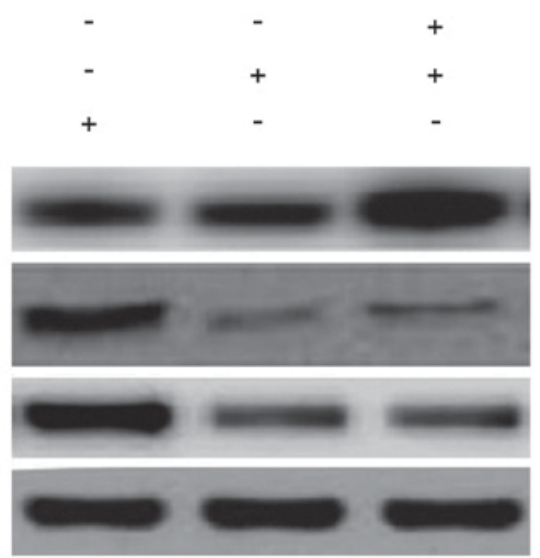

Figure 6. BAG3 promoted the expression of RUNX2 via the upregulation of $\beta$-catenin. (A) SW1353 cells were transfected with control siRNA, BAG3 siRNA, vector or BAG3 overexpression vector for $48 \mathrm{~h}$; GAPDH was used as the loading control. (B) SW1353 cells were transfected with or without control siRNA and $\beta$-catenin siRNA vector for $48 \mathrm{~h}$. The protein expressions of BAG3, $\beta$-catenin and RUNX2 were then determined by western blot analysis; GAPDH was used as the loading control. BAG3, B-cell lymphoma-2 associated athanogene 3; siRNA, small interfering RNA; RUNX2, runt-related transcription factor 2 .

(Table II). Meanwhile, in BAG3 high-expressed chondrosarcoma tissues, the probability of the abnormal expression of $\beta$-catenin was much higher (75.86\%) (Table II). Our statistical analysis showed that there was an obvious correlation between BAG3 and $\beta$-catenin expression in chondrosarcoma patient specimens.

\section{Discussion}

This study was to explore the role of BAG3 in chondrosarcoma and the possible mechanisms for the development of tumorigenesis. BAG3 protein has been found to be associated with anti-apoptotic functions recently. BAG3 gene contained 588 amino acids, located in 10 q25, coded one kind of Bcl-2 related proteins, and could combine with HSP70. Recent studies have shown that BAG3 is upregulated in a variety of malignant tumor, especially adenocarcinoma such as ovarian cancer (16), colon cancer (17), prostate cancer (18), pancreatic cancer (19), breast cancer (20) and thyroid carcinoma (21). Our study revealed the oncogenic roles of BAG3 in chondrosarcoma, suggesting anti-BAG3 might contribute to development of anti-chondrosarcoma drugs. It was confirmed that the expression of BAG3 was correlated with the expression of $\beta$-catenin, and previous studies have proved that the accumulation of $\beta$-catenin promoted the physiological process of Chondrosarcoma (22). Therefore, we can guess that the targeted drugs of BAG3 was studied to reduce the distribution of BAG3 in chondrosarcoma and its expression, which may decrease the accumulation
Table II. Associations between B-cell lymphoma-2 associated athanogene 3 and $\beta$-catenin expression in chondrosarcoma.

\begin{tabular}{|c|c|c|c|c|}
\hline \multirow{2}{*}{$\begin{array}{l}\beta \text {-catenin } \\
\text { expression }\end{array}$} & \multicolumn{2}{|c|}{$\begin{array}{c}\text { BAG3 } \\
\text { expression }\end{array}$} & \multirow[b]{2}{*}{ Chi-square } & \multirow[b]{2}{*}{ P-value } \\
\hline & Low & High & & \\
\hline Normal & 18 & 7 & 7.766 & 0.005 \\
\hline Abnormal & 12 & 22 & & \\
\hline
\end{tabular}

Six was set as the critical value for BAG3 staining score: High expression group, BAG3 expression value $\geq 6$; low expression group, BAG3 expression value $<6$. $\beta$-catenin was judged by the Maruyama standard (12) and was mainly expressed in the cell membrane. The expression of membrane was normal when $\geq 70 \%$ and decreased when $<70 \%$, respectively. The expression of membrane was decreased and abnormal when $\geq 10 \%$ plasma or nuclear expression. BAG3, B-cell lymphoma- 2 associated athanogene 3 .

of $\beta$-catenin in order to achieve low expression level of $\beta$-catenin in chondrocytes, so as to control the occurrence of chondrosarcoma. We found that the BAG3 mRNA and protein expressions were significantly upregulated in chondrosarcoma cells compared with normal cartilage cell line HC-a. Importantly, BAG3 protein expression was upregulated in 59 cases of chondrosarcoma compared with in 30 cases of patients with endogenous chondroma and 8 cases of normal cartilage. We also analyzed BAG3 protein expression levels in chondrosarcoma regarding the patient's clinical pathological parameters, clinical stage and the survival time. Therefore, it is necessary to investigate the functions and mechanisms of BAG3 during the malignant development in chondrosarcoma. We measured the cell proliferation, the colony formation, apoptosis and migration by knocking down or overexpression of BAG3 in chondrosarcoma cells. All these results consistently demonstrated that BAG3 possesses an oncogenic function in chondrosarcoma.

It has been found that $\beta$-catenin as an adhesion factor played an important role in the development of some tumors, such as breast cancer and prostate cancer $(23,24)$. It demonstrated that BAG3 promoted the expression of RUNX2 in chondrosarcoma and that RUNX22 was involve in the migration of multiple bone tumors. Importantly, it was observed that $\beta$-catenin was a key inducing factor of RUNX2 and was upregulated by BAG3 at both mRNA and protein levels. Knockout of $\beta$-catenin in chondrosarcoma cells could not result in high expression of RUNX2 through overexpression of BAG3, indicating that BAG3 upregulated RUNX2 expression through mediating the expression of $\beta$-catenin.

We analyzed 59 cases of chondrosarcoma, 30 cases of naturally express cartilage tumors and 8 cases of normal cartilage tissues using immunohistochemical method and semi-quantitative analysis. Among them, statistical analysis showed that there was an obvious positive correlation between BAG3 and $\beta$-catenin expression, suggesting BAG3 might be involved in the development of chondrosarcoma by regulating $\beta$-catenin clinically. In summary, our study revealed oncogenic 

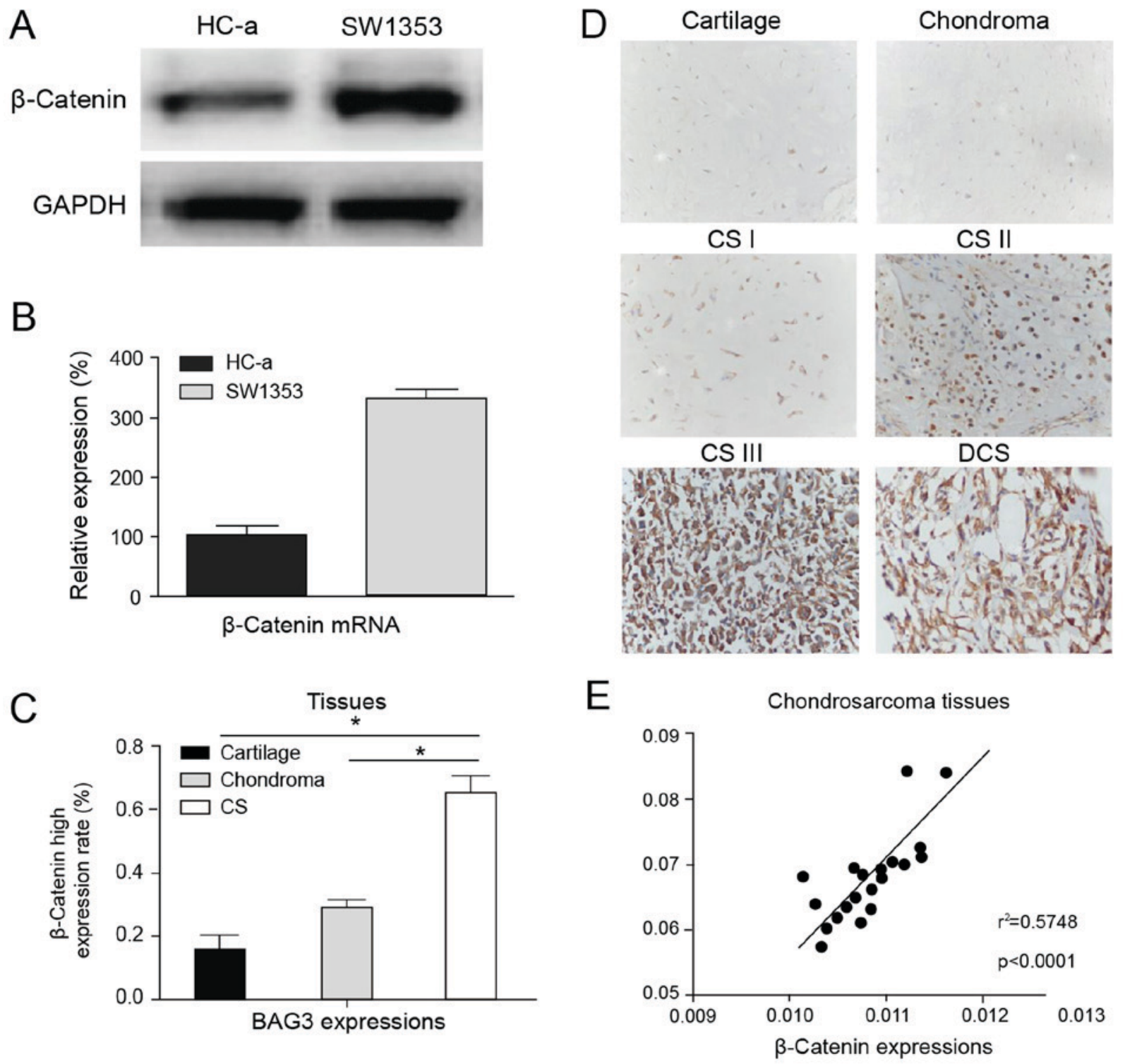

Figure 7. Associations between BAG3 and $\beta$-catenin overexpression in chondrosarcoma. (A) The protein and (B) mRNA expressions of $\beta$-catenin in HC-a and SW1353 cells. (C) The expression of $\beta$-catenin mRNA in human cartilage, chondroma or CS tissues. (D) Immunohistochemical staining of $\beta$-catenin expression in human cartilage tissues, chondroma tissues, CS tumors from grade I to III and DCS. The micrographs shown represent the range of staining observed in tissues (magnification, x200). (E) The association between BAG3 and $\beta$-catenin mRNA levels in 20 CS tissues is presented. Data are presented as the mean \pm standard error of three independent experiments. " $\mathrm{P}<0.05$, as indicated. CS, chondrosarcoma; DCS, dedifferentiated chondrosarcoma; BAG3, B-cell lymphoma-2 associated athanogene 3; siRNA, small interfering RNA.

roles of BAG3 in chondrosarcoma and provided mechanisms that the BAG3-modulated the expression of RUNX2 through upregulation of $\beta$-catenin. This study provides a new foundation for molecule-targeted therapy of human chondrosarcoma.

\section{Acknowledgements}

The authors would like to thank the Department of Pathology (The First Affiliated Hospital of Sun Yat-Sen University, Guangdong, China) for supplying the patients' data.

\section{Funding}

The present study was supported by grants from the National Natural Science Foundation of China (grant nos. 81502327 and 81502021).

\section{Availability of data and materials}

All data generated and analyzed during this study are included in this published article.

\section{Authors' contributions}

WC, YD, XL and WZ collected the data. HS conducted the experiments, and drafted the manuscript and revised it critically for important intellectual content. LW analyzed and interpreted the data.

\section{Ethics approval and consent to participate}

Patient's written informed consent and approval from the Institute Research Ethics Committee of The First Affiliated 
Hospital of Sun Yat-Sen University (Guangzhou, China) were obtained (Ethics Committee approval no. 201301).

\section{Consent for publication}

All patients provided written informed consent.

\section{Competing interests}

The authors declare that they have no competing interests.

\section{References}

1. Gelderblom H, Hogendoorn PC, Dijkstra SD, van Rijswijk CS, Krol AD, Taminiau AH and Bovée JV: The clinical approach towards chondrosarcoma. Oncologist 13: 320-329, 2008.

2. Xu J, Li D, Xie L, Tang S and Guo W: Mesenchymal chondrosarcoma of bone and soft tissue: A systematic review of 107 patients in the past 20 years. PLoS One 10: e0122216, 2015.

3. Wagner MJ, Livingston JA, Patel SR and Benjamin RS: Chemotherapy for bone sarcoma in adults. J Oncol Pract 12: 208-216, 2016.

4. Onishi AC, Hincker AM and Lee FY: Surmounting chemotherapy and radioresistance in chondrosarcoma: Molecular mechanisms and therapeutic targets. Sarcoma 2011: 381564, 2011.

5. Italiano A, Mir O, Cioffi A, Palmerini E, Piperno-Neumann S, Perrin C, Chaigneau L, Penel N, Duffaud F, Kurtz JE, et al: Advanced chondrosarcomas: Role of chemotherapy and survival. Ann Oncol 24: 2916-2922, 2013.

6. Rosati A, Graziano V, De Laurenzi V, Pascale M and Turco MC: BAG3: A multifaceted protein that regulates major cell pathways. Cell Death Dis 2: e141,2011.

7. Fuchs M, Poirier DJ, Seguin SJ, Lambert H, Carra S, Charette SJ and Landry J: Identification of the key structural motifs involved in HspB8/HspB6-Bag3 interaction. Biochem J 425: 245-255, 2009.

8. Li X, Colvin T, Rauch JN, Acosta-Alvear D, Kampmann M, Dunyak B, Hann B, Aftab BT, Murnane M, Cho M, et al: Validation of the Hsp70-Bag3 protein-protein interaction as a potential therapeutic target in cancer. Mol Cancer Ther 14: 642-648, 2015

9. Colvin TA, Gabai VL, Gong J, Calderwood SK, Li H, Gummuluru S, Matchuk ON, Smirnova SG, Orlova NV, Zamulaeva IA, et al: Hsp70-Bag3 interactions regulate cancer-related signaling networks. Cancer Res 74: 4731-4740, 2014.

10. Kong DH, Zhang Q, Meng X, Zong ZH, Li C, Liu BQ, Guan Y and Wang HQ: BAG3 sensitizes cancer cells exposed to DNA damaging agents via direct interaction with GRP78. Biochim Biophys Acta 1833: 3245-3253, 2013.

11. Habata S, Iwasaki M, Sugio A, Suzuki M, Tamate M, Satohisa S, Tanaka R and Saito T: BAG3-mediated Mcl-1 stabilization contributes to drug resistance via interaction with USP9X in ovarian cancer. Int J Oncol 49: 402-410, 2016.
12. Maruyama K, Ochiai A, Akimoto S, Nakamura S, Baba S, Moriya Y and Hirohashi S: Cytoplasmic beta-catenin accumulation as a predictor of hematogenous metastasis in human colorectal cancer. Oncology 59: 302-309, 2000.

13. Komori T: Regulation of skeletal development by the Runx family of transcription factors. J Cell Biochem 95: 445-453, 2005.

14. Banerjee C, McCabe LR, Choi JY, Hiebert SW, Stein JL, Stein GS and Lian JB: Runt homology domain proteins in osteoblast differentiation: AML3/CBFA1 is a major component of a bone-specific complex. J Cell Biochem 66: 1-8, 1997.

15. Lemieux E, Cagnol S, Beaudry K, Carrier J and Rivard N: Oncogenic KRAS signalling promotes the Wnt/ $\beta$-catenin pathway through LRP6 in colorectal cancer. Oncogene 34: 4914-4927, 2015.

16. Suzuki M, Iwasaki M, Sugio A, Hishiya A, Tanaka R, Endo T, Takayama S and Saito T: BAG3 (BCL2-associated athanogene 3) interacts with MMP-2 to positively regulate invasion by ovarian carcinoma cells. Cancer Lett 303: 65-71, 2011.

17. Shi H, Xu H, Li Z, Zhen Y, Wang B, Huo S, Xiao R and Xu Z: BAG3 regulates cell proliferation, migration and invasion in human colorectal cancer. Tumour Biol 37: 5591-5597, 2016.

18. Staibano S, Mascolo M, Di Benedetto M, Vecchione ML, Ilardi G, Di Lorenzo G, Autorino R, Salerno V, Morena A, Rocco A, et al: BAG3 protein delocalisation in prostate carcinoma. Tumour Biol 31: 461-469, 2010.

19. Falco A, Rosati A, Festa M, Basile A, De Marco M, d'Avenia M, Pascale M, Dal Piaz F, Tavano F, Di Mola FF, et al: BAG3 is a novel serum biomarker for pancreatic adenocarcinomas. Am J Gastroenterol 108: 1178-1180, 2013.

20. Felzen V, Hiebel C, Koziollek-Drechsler I, Reißig S, Wolfrum U, Kögel D, Brandts C, Behl C and Morawe T: Estrogen receptor a regulates non-canonical autophagy that provides stress resistance to neuroblastoma and breast cancer cells and involves BAG3 function. Cell Death Dis 6: e1812, 2015.

21. Chiappetta G, Ammirante M, Basile A, Rosati A, Festa M, Monaco M, Vuttariello E, Pasquinelli R, Arra C, Zerilli M, et al: The antiapoptotic protein BAG3 is expressed in thyroid carcinomas and modulates apoptosis mediated by tumor necrosis factor-related apoptosis-inducing ligand. J Clin Endocrinol Metab 92: 1159-1163, 2007.

22. Chen C, Zhou H, Zhang X, Ma X, Liu Z and Liu X: Elevated levels of Dickkopf-1 are associated with beta-catenin accumulation and poor prognosis in patients with chondrosarcoma. PLoS One 9: e105414, 2014

23. Nowicki A, Sporny S and Duda-Szymanska J: $\beta$-catenin as a prognostic factor for prostate cancer $(\mathrm{PCa})$. Central European $\mathrm{J}$ Urol 65: 119-123, 2012.

24. Calaf GM, Alvarado ME and Hei TK: Beta catenin is associated with breast cancer progression in vitro. Int J Oncol 26: 913-921, 2005. 